Piglets weaned at the mean age of 26 days $(6.5 \mathrm{~kg})$ were fed ad libitum for two weeks the 1 st-age diet already used before weaning, then for 28 or 35 days (from 9 to $25 \mathrm{~kg}$ live weight) the experimental diets in the form of meal or pellets.

The most remarkable and regular effect of pellets was an improvement of the feed efficiency (4.9 p. 100 reduction in the feed conversion ratio) by reducing the level of voluntary feed intake $(-4$ p. 100$)$ without noticeably affecting the growth rate $(+1.1$ p. 100). The nature of the cereal did not modify this effect which was similar during the first two weeks and during the whole experiment.

This improvement was slightly lower than that obtained in most of the works quoted in the literature. Nevertheless it is interesting from an economic point of view as long as the cost of pellets is 105 p. 100 lower than that of meals.

\title{
Effect of two hypocalcemic diets on the absorption and retention of calcium and phosphorus in the growing pig
}

\author{
A. POINTILLART, Nicole FONTAINE \\ I.N.R.A., Station de Recherches de Nutrition, 78350 Jouy-en-Josas \\ France
}

Three 10-day balance experiments were carried out separately in 4 growing pigs which had already received a low calcium $(0.1 \mathrm{p} .100)$, a high phosphorus $(1.2 \mathrm{p} .100)$ or a control $(0.9$ p. $100 \mathrm{Ca}$ and 0.8 p. $100 \mathrm{P})$ diet for 6 weeks. Additional data concerning bone metabolism or hormonal regulation (PTH and CT) from other experiments are also presented in this report.

Only the calcium deficient diet reduced the growth rate and produced apparent bone disturbances (deformation of snout). Phosphorus absorption was directly correlated with phosphorus intake whereas calcium intake had no influence on $\mathrm{P}$ absorption. The same phenomenon was also observed for calcium. In spite of a temporary (high $\mathrm{P}$ diet) or steady (low $\mathrm{Ca}$ diet) hypocalcemia, no hyperparathyroidism was observed. Low $\mathrm{Ca}$ and high $\mathbf{P}$ diets decreased plasma CT levels. These diets also decreased the phosphorus retention (20.4 p. $100 \pm 2$ and 36.5 p. $100 \pm 2.4$, respectively) compared with that of the control diet (46.3 p. $100 \pm 1.4)$ and produced a high phosphaturia. Relative $\mathrm{Ca}$ absorption did not change whatever the diet (about $50 \mathrm{p}$. 100 of the intake). However, low $\mathrm{Ca}$ diet decreased urinary $\mathrm{Ca}$ excretion. Nephrocalcinosis appeared with the high $\mathrm{P}$ diet. The low Ca diet caused some evidences of hyperosteolysis such as a high urinary hydroxyproine and a higher percentage of bone marrow spaces. However, some of the data concerning. bone mineral content (BMC) are in contrast with the above results. Moreover, in some cases BMC indicated a stimulated bone resorption with the high $\mathrm{P}$ diet. Discrepancies. between the results obtained for different criteria (BMC, plasma minerals and hormones, balances, etc.) are discussed. In conclusion, as for other species excess in phosphorus is not recommended for pigs. 